\title{
Les Trois Mousquetaires : enjeux d'une réappropriation dans Milady de Winter d'Agnès Maupré Suivi d'un Entretien avec Agnès Maupré
}

\author{
Laboratoire SLAM, Université d'Évry \\ "Qui dira les ravages exercés par la femme imaginaire - ange ou madone - qui \\ gouverne le siècle? Mais si les héroïnes du XIXe siècle n'ont pas fini de nous \\ solliciter en dépit des changements profonds intervenus depuis dans les mours, si \\ elles revivent si souvent sur nos écrans, $n^{\prime} e s t-c e$ pas précisément par leur soif de \\ bonheur, leurs contradictions et le destin qui les broie? La liberté ne se \\ partageant pas, aucune de leurs aspirations ne saurait nous être étrangère. »
}

Sophie Bonadè

(Stéphane Michaud 152)

\section{Introduction}

Cet article aborde une œuvre écrite par une femme, prenant une femme pour personnage principal: Milady de Winter, une bande dessinée d'Agnès Maupré (2010 et 2012). Les deux tomes qui la composent (respectivement 160 et 141 pages) ont pour personnage principal Milady, un personnage des Trois mousquetaires d'Alexandre Dumas. Le récit est en noir et blanc, dessiné à la plume, et les ombres sont réalisées en lavis de gris.

Il s'agira ici d'étudier l'œuvre d'Agnès Maupré sous l'angle du genre, c'est-àdire des rapports de sexes qui se jouent dans cette bande dessinée. Les conflits genrés, présents dans l'ouvrage Les Trois mousquetaires, sont mis en exergue dans Milady de Winter par l'interversion qu'opère Agnès Maupré entre les protagonistes masculins, qui deviennent des personnages secondaires, et Milady de Winter qui devient le personnage principal. Cette inversion des rôles crée une nouvelle lecture de l'histoire créée par Alexandre Dumas où la question des rapports de sexes devient primordiale.

Deux inversions présentes dans Milady de Winter segmentent cet article. La première est la relecture du personnage de Milady comme victime des hommes avant de devenir une criminelle, et la seconde est le glissement de la figure de la femme fatale vers celle de la femme en lutte. Pour réaliser cet article, je me suis entretenue par échange de courriels avec Agnès Maupré, reproduit en annexe.

\section{Contexte}


La bande dessinée n'a pas dérogé à la règle de la suprématie masculine. L'arrivée, durant ces dernières années, d'une vague de femmes a permis d'atteindre les $12 \%$ d'auteures ${ }^{1}$, mais leur présence reste cependant discrète. ${ }^{2}$

Dans les années 1970, Claire Bretécher et Chantal Montellier publient des bandes dessinées où elles explorent le thème des femmes et leurs droits (Claire Bretécher avec Cellulite et Chantal Montellier avec Odile et les crocodiles). Ces auteures font figure d'exceptions, tant le nombre de femmes est faible dans ce milieu $^{3}$. Leur travail, qui s'inscrit dans les prémices de l'émergence de la bande dessinée d'auteurs qui aura lieu dans les années 1990 (Boy 5-9), permet de faire reconnaître les femmes comme auteures.

Durant les années 1990 ont lieu plusieurs changements dans l'univers de la bande dessinée qui préparent le terrain d'une plus grande représentativité des femmes. L'année 1990 voit d'abord se créer L'Association, un collectif d'auteurs réunis dans une structure associative. Leur souhait est de publier une bande dessinée différente de celle publiée par les gros éditeurs ${ }^{4}$. Leurs publications, toujours en noir et blanc, se concentrent autour des thèmes de l'intime. Avant 2000, L'Association ne publie que peu de bandes dessinées créées par des femmes, et la grande majorité de leur catalogue reste, jusqu'à aujourd'hui, constituée d'auteurs masculins. Cette structure apporte néanmoins un public nouveau à la bande dessinée, intéressé par de nouveaux types de récits. La création de cette nouvelle structure éditoriale, suivie par d'autres (Amok, Acte Sud BD, 6 pieds sous terre et Les Éditions de l'an 2) permet à de nombreux auteurs (Joan Sfar, Lewis Trondheim, David B, Edmond Baudoin) et auteures (Marjane Satrapi, Lisa Mandel) d'émerger.

${ }^{1}$ Lors de l'interview d'Agnès Maupré., nous lui avons demandé si elle préférait être appelée « auteur », « auteure » ou « autrice ». Sa réponse a été qu'« auteur » et « auteure » lui convenaient bien. Nous avons choisi d'utiliser « auteure », car ce terme nous permet d'éviter la répétition des termes « auteur femme » ou « auteur féminin ».

2 Sur la question de la proportion (Groensteen, «Femme (2) »). Signalons tout de même que le questionnaire de l'enquête « Auteurs 2016 » adressé à tous les auteurs de bande dessinée, professionnels ou amateurs, par les États Généraux de la Bande Dessinée, a été rempli par $27 \%$ de femmes.

${ }^{3} \mathrm{Si}$ les auteures sont moins nombreuses, l'histoire de l'art a en outre davantage tendance à les invisibiliser. Comme le rapporte Irène Le Roy Ladurie, Jessica Kohn a rappelé durant la table ronde "Bande dessinée : où sont les femmes ? " que si l'histoire de la bande dessinée recensait 1\% d'auteures d'illustrés dans les années 1950, les femmes représentaient en fait $10 \%$ de la profession.

${ }^{4}$ Il faut cependant savoir que l'Association a refusé de publier le travail de Chantal Montellier car il le trouvait d'un militantisme trop poussif et démonstratif. (Boy 94). 
Un autre événement permet de faire reconnaitre les femmes comme auteures légitimes de bande dessinée. En 2000, L'Association publie le premier tome de Persépolis de Marjane Satrapi. Cette bande dessinée reste, à ce jour, le plus grand succès de l'éditeur. Elle permet la reconnaissance de la bande dessinée dite «d'auteurs » et impose une femme auteure. La période semble plus ouverte à l'idée de femmes auteures et de nouvelles créatrices de bande dessinée se font connaître, telles que Pénélope Bagieu, Margaux Motin, Marion Montaigne et Tanxxx.

Parmi ces nouvelles auteures, se trouve Agnès Maupré. Après un an aux Beaux-arts d'Angoulême, elle s'inscrit aux Beaux-arts de Paris. Quand, en 2010, parait le premier tome de Milady de Winter, elle a déjà publié trois albums : Les Contes du chat perché (2 tomes) (Aymé et Maupré) et Petit traité de morphologie (Maupré).

\section{Archéologie du personnage : qui est Milady de Winter?}

Le personnage de Milady de Winter est, aujourd'hui encore, célèbre dans l'imaginaire occidental, 150 ans après sa création. Ce n'est cependant pas tant la femme présente dans le roman Les Trois mousquetaires qui est connue, que ses avatars cinématographiques depuis la première adaptation en 1903 par Georges Méliès (Les Mousquetaires de la reine). Depuis lors, le roman d'Alexandre Dumas a été adapté plus d'une vingtaine de fois pour le grand écran et une dizaine de fois pour la télévision. Ces œuvres ont comme point commun d'être plus ou moins fidèles au roman original. La longueur de celui-ci (plus de 800 pages) a incité les scénaristes à élaguer l'histoire d'Alexandre Dumas pour obtenir des scénarios d'une durée d'environ deux heures. Les histoires des personnages principaux (les mousquetaires et le Cardinal de Richelieu) ne pâtissent que rarement de ces coupes $^{5}$, mais de nombreux personnages secondaires sont simplifiés ou purement supprimés.

Le passé de Milady est ainsi souvent effacé, pour ne laisser apparaître à l'écran que la figure de "femme fatale », que le cinéma aime tant (Doane). À cette première transformation du personnage de Milady s'ajoute le choix des actrices qui l'interprètent. Lana Turner dans la version de 1948 (The Three Musketeers), Yvonne Sanson dans celle de 1953 (Les trois mousquetaires), Faye Dunaway en 1974 (The Three Musketeers) sont des actrices connues à

${ }^{5}$ Cette affirmation ne peut pas être appliquée au dernier film réalisé à propos de l'ouvrage d'Alexandre Dumas : The Three Musketeers de Paul W. S. Anderson. Ce film ne respecte aucunement le récit original. Pour exemple, dans ce film, le Comte de Buckingham, interprété par Orlando Bloom, commande à une flotte de bateaux volants. 
HollywAnood pour leurs interprétations de femmes séductrices - Lana Turner dans Le Facteur sonne toujours deux fois (The Postman Always Rings Twice, 1946), Yvonne Sanson dans Le Crime de Giovanni Episcopo (Il delitto di Giovanni Episcopo, 1947) et Faye Dunaway dans L'Affaire Thomas Crown (The Thomas Crown Affair, 1968). Ce choix d'actrices finit d'imposer Milady de Winter comme femme dangereuse, séductrice et calculatrice, comme «femme fatale », en somme.

C'est en utilisant son passé malheureux, si souvent occulté, que l'auteure Agnès Maupré a commencé son récit, expliquant par-là l'évolution du personnage, prenant le contre-pied des adaptations cinématographiques : « Les adaptations que j'ai pu voir, c'était quand j'étais petite, et c'est plutôt ce qui m’avait donné envie de ne pas lire Les Trois Mousquetaires, donc je n'ai pas essayé de les revoir. »(Berthelon)

Dans Les Trois mousquetaires, Milady est présentée au lecteur dès le premier chapitre lorsqu'elle croise par hasard la route de d'Artagnan. Durant la première partie du récit, elle n'est qu'un personnage secondaire. À la moitié du roman, elle suscite l'intérêt amoureux du jeune d'Artagnan, avant d'être l'ennemie principale des mousquetaires. C'est durant cette seconde partie que le passé de Milady est raconté par bribes.

Avant d'être Milady de Winter, elle était religieuse sous le nom d'Anne de Breuil. Durant cette période, elle a entretenu une relation avec un jeune prêtre le récit précise que c'est Milady, alors âgée de 13 ans, qui a séduit le prêtre - avec qui elle décide de s'enfuir. Condamnés pour vol et pour avoir rompu leurs vœux, ils sont tous deux marqués de la Fleur de $\operatorname{Lys}^{6}$ par le bourreau de Lille, qui est aussi le frère du prêtre. Ils s'échappent ensuite et partent vivre dans le Berry où ils se font passer pour frère et sœur et vivent dans la misère. Un jour, Anne de Breuil rencontre le Comte de la Fère - que le lecteur connaît sous le nom d'Athos - qui tombe amoureux d'elle et l'épouse. Anne de Breuil devient la Comtesse de la Fère. À la suite de cette trahison, le prêtre se suicide. Un jour Athos découvre le Lys marqué sur son épaule, et la pend alors à un arbre. Elle survit à cet événement et, profitant de ce qu'on la croit morte, part pour l'Angleterre où elle devient Milady et épouse Lord de Winter. À la mort de celuici - dont le frère accuse Milady de l'avoir empoisonné -, elle retourne en France où elle devient espionne du cardinal.

Le récit d'Alexandre Dumas commence quelque temps après. Milady n'est cependant pas au bout de ses peines puisqu'elle est violée par d'Artagnan durant

${ }^{6} \mathrm{La}$ flétrissure était une peine infligée à certains criminels. Leur peau était marquée au fer rouge avec le motif d'une fleur de Lys. 
le roman (Dumas 512-513), et qu'elle finit jugée par un tribunal fantoche, composé des mousquetaires et du beau-frère de Milady, accusateurs et juges, qui la condamnent à mort et la font exécuter par le bourreau de Lille. La fin du roman est donc l'accomplissement d'une vengeance des mousquetaires envers Milady, consécutive au meurtre de Constance, la maittresse de D'Artagnan, par Milady.

Milady apparait donc comme un personnage dont le destin est entièrement régi par des hommes. Toutes ses tentatives d'action en son nom propre, telle sa vengeance contre les mousquetaires, sont contrecarrées. Elle est inévitablement renvoyée à son statut de femme dans un monde d'hommes, ou à celui de personnage féminin dans un livre d'homme $(s)^{7}$.

Il est difficile d'évaluer le degré de misogynie de roman d'Alexandre Dumas sans questionner toute la production artistique et culturelle du siècle où il a été écrit. Les Trois mousquetaires est en effet un roman inscrit dans son époque, c'està-dire un siècle qui a entendu les revendications des femmes révolutionnaires et qui a assisté à l'échec de leurs combats pour leurs droits : «Exaltées comme mères au secret du foyer, les femmes font les frais d'une volonté restauratrice. La Révolution a pu déposer le roi et inventer le citoyen ; elle n'a pas créé la citoyenne. » (Michaud 149)

Stéphane Michaud estime en outre que la production artistique du XIXe siècle est hantée par l'image des femmes, c'est-à-dire par l'idée que les artistes, souvent masculins, se font d'elles, au détriment de personnages féminins plus réalistes :

Quelle est donc cette force qui l'emporte sur les idéologies et exclut la femme du registre des faits ? Impossible de croire que ce soit la Nature comme l'époque l'avance avec une téméraire insistance. C'est celle des images. La femme ici est imaginaire. Idole, elle fascine le siècle (147).

Milady est une de ces «femmes images», devenue l'archétype de la femme dangereuse ou « femme fatale $»^{8}$ sur lequel nous reviendrons.

${ }^{7}$ Par l'expression « livre d'homme(s) », nous entendons un livre écrit par un homme et dont les principaux personnages sont des hommes. Le sexe des lecteurs des romansfeuilletons est difficile à déterminer, ceux-ci étant parfois lus dans des lectures publiques.

${ }^{8}$ Selon la typologie établit par Christopher Vogler dans Le guide du scénariste: la force d'inspiration des mythes pour l'écriture cinématographique ù.et romanesque, Milady serait le personnage protéiforme, c'est-à-dire un personnage incertain, car le héros ne connaît pas ses motivations. 
L'auteur donne également une place à un plusieurs personnages féminins (la Reine, Constance Bonacieux, la Duchesse de Chevreuse) qui, sans avoir l'importance des personnages masculins, agissent et participent à l'intrigue. Le romancier crée cependant ses personnages selon une distinction essentielle entre homme et femme, teintée d'une certaine misogynie. Il y a dans Les Trois mousquetaires une nature féminine et une nature masculine auxquelles les individus doivent se soumettre, caractéristique de la production artistique de l'époque :

Mais la France manifeste peut-être plus nettement et plus tôt qu'aucun autre pays à quel point toute une part de la floraison artistique et intellectuelle s'alimente à une représentation sexuée des choses qui fixe pour but à l'homme la domination de l'inquiétante étrangeté féminine. (Michaud 161)

Toute tentative d'empiéter sur le territoire de l'autre sexe, surtout du masculin?', est sévèrement jugée. Milady subit ce sort, comme le montre Agnès Maupré : "Les mousquetaires sont quatre. Elle est seule. Ses actes sont condamnés plus durement que leurs équivalents commis par des hommes. Les mousquetaires sont des soldats, ils sont censés se battre. Chez elle, c'est du vice. » (Bonadè).

Milady est, dans l'œuvre de Dumas, une femme solitaire sans cesse aux prises avec des hommes qui finissent par triompher d'elle. Elle est, de plus, présentée comme une femme diabolique qui outrepasse les limites de son sexe. Agnès Maupré, dans Milady de Winter, propose aux lecteurs une nouvelle version du personnage. Cette réécriture est évidemment permise par la partielle reconnaissance, à partir de la seconde partie du XXe siècle, des violences faites aux femmes, ainsi que de la domination sociale masculine. Ces deux sujets sont d'ailleurs inclus dans la réécriture du personnage de Milady de Winter.

\section{Milady, victime de la domination masculine}

Les Trois mousquetaires est un roman publié entre mars et juillet 1844. Son intrigue se situe cependant au XVIIe siècle (de 1625 à 1627). Alexandre Dumas se plait, à plusieurs reprises, à rappeler les différences de mœurs entre le XVIIe et le XIXe siècle, particulièrement dans les rapports de classes et de sexes.

Milady de Winter d'Agnès Maupré fait donc se superposer trois époques

${ }^{9}$ L'empiétement du masculin sur le féminin est moins sévèrement réprimandé. Les mousquetaires Porthos et Aramis sont parfois moqués pour leur trop grand attachement à leur apparence physique ou leur trop grand romantisme (Dumas, «L'antichambre» 32-45) mais ils ne subissent pas d'autre conséquence. 
(XVIIe, XIXe et XXIe siècles), au sein desquelles les rapports sociaux des sexes sont très différents. Contrairement à Dumas, elle n'émet pas de discours explicite sur les siècles précédents, mais met en scène, frontalement et graphiquement (Figures 1, 2 \& 3), les violences des hommes sur Milady, présentes dans le roman original. Alors que Les Trois mousquetaires ne s'attardait pas sur ces violences, le premier tome de Milady de Winter s'ouvre sur une image de Milady pendue à un arbre (Figure 1), faisant de cet événement le départ des aventures du personnage.

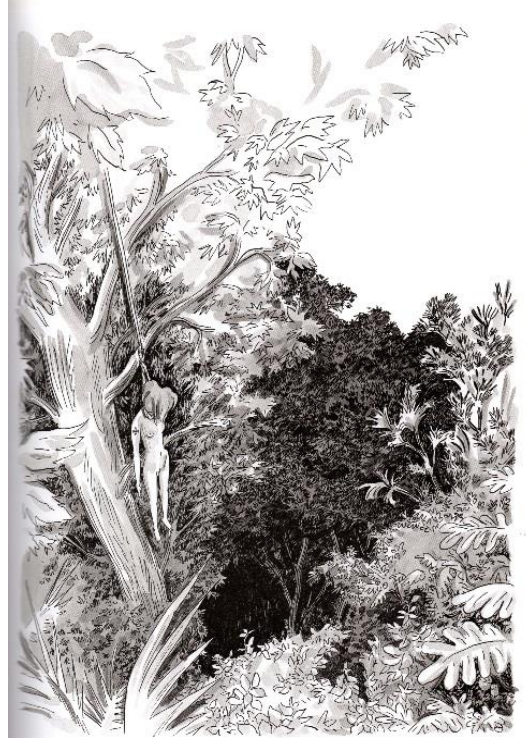

Figure 1 : Milady pendue par son mari @Maupré/Ankama Éditions

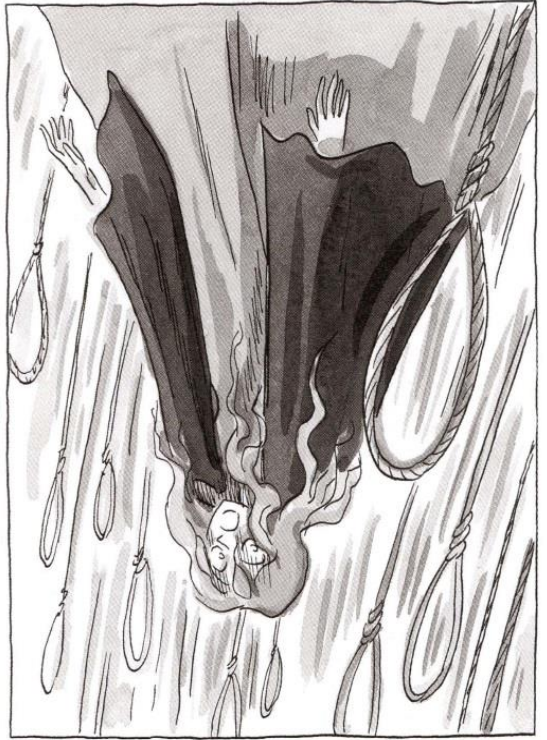

Figure 2: Les cauchemars qui hantent Milady par la suite. CMaupré/Ankama 


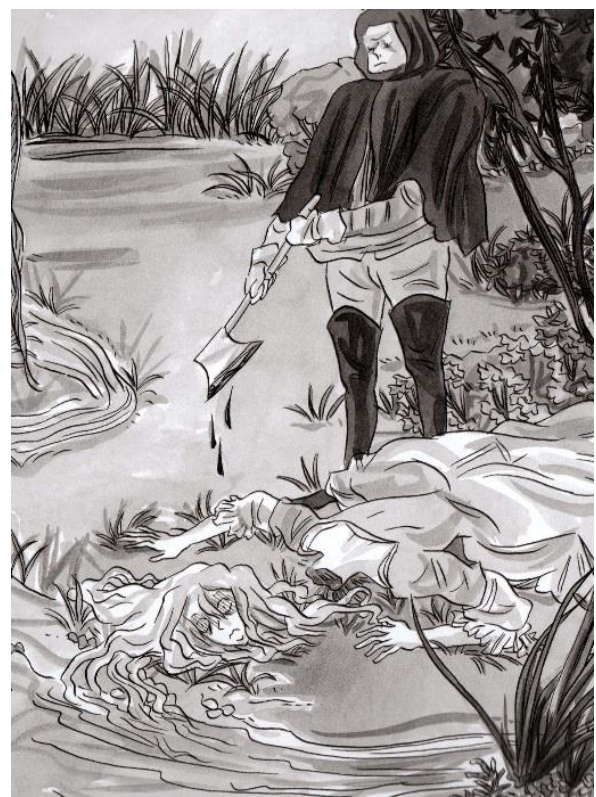

Figure 3. La mort de Milady décapitée par le Bourreau de Lille. CMaupré/Ankama Éditions

L'auteure renverse ainsi le point de vue de la narration en faisant de Milady le personnage principal de son récit, ce qui permet au lecteur d'adopter plus aisément son point de vue et de la considérer en tant que victime.

Les violences qu'elle subit ont une résonnance aujourd'hui, car elles sont aujourd'hui connues - même si elles ne sont pas toujours dénoncées ni jugées : viol, tentative de meurtre par un conjoint, assassinat (Romito 11-28). Milady de Winter vit l'expérience des différentes violences physiques liées à la domination masculine.

Si Milady est présentée comme une victime, elle apparait également comme une femme qui a survécu à cette violence et qui a été façonnée par celles-ci. Les expériences de Milady peuvent ainsi être lues en regard de ce que peut vivre chaque femme qui se construit dans une société dominée par sa moitié de population masculine (Delphy 55-90 ; Stoltenberg 33-51). Comme le précise Agnès Maupré, ce ne sont pas seulement les violences physiques subies qui sont abordées, mais aussi une expérience de lutte au quotidien pour sa reconnaissance et sa place :

Même quand une femme ne subit aucune violence dans sa vie, il y a des moments où elle ressent son infériorité physique par rapport aux hommes, le soir, dans des rues sombres ou autre. Milady a vécu un copieux échantillonnage de ce qui peut arriver de plus brutal à une femme. Elle rebondit, elle en fait sa force, mais cette force la rend seule de son espèce. (Bonadè) 
Agnès Maupré prend ainsi le contre-pied du roman d'Alexandre Dumas, qui ne s'attachait que très rarement à dénoncer les méfaits de ses personnages, ou, quand il le faisait, les relégitimait aussitôt.

En effet, l'un des aspects les plus sexistes de l'œuvre d'Alexandre Dumas réside dans la banalisation des sévices que les hommes font subir aux femmes, particulièrement à Milady. Par exemple, Milady, qui est amoureuse du Comte de Wardes, lui écrit plusieurs lettres interceptées par d'Artagnan. Le mousquetaire se fait passer pour le Comte de Wardes et répond aux lettres en acceptant la proposition de rendez-vous fixée par Milady. Le soir venu, il se glisse dans la chambre de Milady, alors que toutes les lumières sont éteintes, et passe la nuit avec elle. Si l'aspect sexuel de cette rencontre n'est pas décrit, il est fortement sous-entendu (Dumas 512-513). Cette scène décrit donc un viol, puisque Milady pense offrir son corps à un homme, et qu'il s'agit en fait d'un autre, qui abuse d'elle.

Le roman fait quelques fois référence aux remords de d'Artagnan (Dumas 522), mais ce fait est toujours suivi par une comparaison des crimes de d'Artagnan et de ceux de Milady, qui joue en défaveur de cette dernière. On assiste ainsi à un double mouvement de dénonciation et de justification, où les actes des mousquetaires sont mis en lumière pour être aussitôt excusés par le romancier. Cette technique trouve son apogée dans le procès de Milady, conduit par les mousquetaires eux-mêmes, durant lequel ceux-ci la forceront à accepter leurs excuses et lui en présenteront avant de l'exécuter : « Et moi, dit d'Artagnan, pardonnez-moi, madame, d'avoir, par une fourberie indigne d'un gentilhomme, provoqué votre colère; et, en échange, je vous pardonne le meurtre de ma pauvre amie et vos vengeances cruelles pour moi, je vous pardonne et je pleure sur vous. Mourez en paix. » (Dumas 882)

Cette technique est également utilisée pour justifier la tentative de meurtre d'Athos sur sa femme. Lorsqu'il raconte pour la première fois son histoire à d'Artagnan, celui-ci est choqué par sa violence (Dumas 421). Cependant, cela ne l'empêche pas de conserver toute son amitié à Athos et de prendre son parti, dès qu'il apprend que la femme d'Athos était Milady. Là encore, les actes criminels contre Milady sont justifiés par son statut de femme meurtrière.

Ce discours de légitimation des violences contre une femme trouve un autre écho pour un lecteur et une lectrice contemporains. Depuis les années 1970, les travaux menés sur les violences faites aux femmes ont mis en lumière les différents moyens de justification utilisés par les coupables (Romito). Ces études ont aussi dénoncé les différentes techniques utilisées pour réduire les femmes 
battues au silence : légitimation des bourreaux donc, mais aussi culpabilisation et absence de tout soutien législatif et juridique pour les victimes. Milady est, dans Les Trois mousquetaires, une femme qu'on a fait taire. Elle ne pourrait obtenir de soutien juridique puisqu'elle est marquée et considérée comme une criminelle. De plus, dans le roman, elle est l'épouse et la sujette d'Athos, qui est le seigneur des terres où il réside. Athos possède donc un double pouvoir de vie et de mort sur Milady, qui n'est jamais questionné dans l'œuvre d'Alexandre Dumas.

Milady de Winter est un personnage féminin auquel Alexandre Dumas ne laisse que très rarement la parole, s'inscrivant ainsi dans son siècle comme que décrit Stéphane Michaud, citant Yves Bonnefoy :

Il faudra en vérité attendre les travaux de la critique la plus récente pour qu'éclate au grand jour l'incroyable mauvaise foi dont fait preuve la littérature, plus redoutable sans doute à la fin du siècle qu'à ses débuts. Pour reprendre les termes de l'un des meilleurs observateurs de la période, les exemples sont légion dans lesquels «les femmes, qui sont victimes, semblent en fait coupables, et n'ont pas de mots pour se faire entendre » (Yves Bonnefoy, La Vérité de la parole). (Michaud 125)

Milady de Winter détruit le processus de dénonciation et justification en focalisant le récit sur la victime. Milady - et le lecteur avec elle - peut difficilement pardonner à quelqu'un qui ne s'est jamais repenti et n'a jamais essayé de se racheter. Agnès Maupré insiste sur le fait que Milady, avant d'être une femme meurtrière, est une femme qui a subi des violences. Ceci est chronologiquement vrai dans Les Trois mousquetaires puisque le premier meurtre - présumé - de Milady est celui de Lord de Winter, son second époux après Athos. Il aurait donc eu lieu après la pendaison de Milady par Athos. Milady, en tous les cas, ne connaît aucun recours à la violence qui lui est infligée. Elle est victime d'hommes, de son mari Athos, de d'Artagnan, et de l'état qui, incarné par Richelieu, ne lui apporte aucun secours. En tant qu'auteure, Agnès Maupré redonne au personnage de Milady, la parole qui fait si souvent défaut aux femmes battues.

\section{De la femme fatale à la femme en lutte}

Après s'être concentrée sur les sévices subis, l'auteure présente les réponses de son personnage à ces violences. L'existence du personnage de Milady dans le roman Les Trois mousquetaires était à l'époque symbole d'un bouleversement dans les rapports entre les sexes. Les femmes s'étaient mobilisées pour obtenir des droits durant la révolution, et continuaient à s'organiser. Milady apparaît donc comme une femme capable de vivre sans hommes, mais aussi une femme dangereuse et meurtrière. Son personnage peut ainsi être lu comme une peur de l'émancipation féminine ; elle est le rappel du risque que courent les hommes (maris, amants ou hommes d'État) s'ils ont à leurs côtés une femme qui ne se soumet pas à la domination masculine. Le personnage de Milady incarne le 
backlash de Susan Faludi c'est-à-dire la revanche ou le retour de bâton qui guette toute minorité après une période d'émancipation et d'obtention de nouveaux droits : après les barricades le retour au foyer.

Or, en personnifiant cette angoisse masculine de l'émancipation féminine, Milady s'apparente à la figure de la « femme fatale », dont Mary Ann Doane dira dans Femmes fatales: Feminism, Film Theory, Psychoanalysis : "Hence, it would be a mistake to see her as some kind of heroine of modernity. She is not the subject of feminism but a symptom of man fears about feminism. » (2)

L'expression «femme fatale » apparaît au début du XXe siècle, mais cette figure hante l'art depuis bien plus longtemps. Les différents ouvrages traitant de la «femme fatale» s'accordent sur les contours flous de sa définition (Braun, Doane, Dottin-Orsini, Grandordy), mais tentent tout de même de la caractériser. Ainsi la «femme fatale » est la femme dangereuse pour l'homme, qu'elle séduit et détruit :

La femme fatale n'est pas seulement la femme qui tue. Elle se confond également avec la mégère, version peu décorative, mais redoutable de celle qui gâche une vie d'homme, avec la dépravée à l'immoralité contagieuse, avec la trop belle au néfaste pouvoir. » (Dottin-Orsini 17)

C'est une femme, ou un personnage, usuellement féminin, dont le comportement conscient ou inconscient et vise à amener l'homme à sa déchéance ou à sa perte ou à le placer dans une situation humiliante. La Femme fatale use habituellement de séduction, et dans ses formes les plus modernes au XXIe siècle, tend à user d'agressivité, mais pas que dans ses formes modernes. L'agressivité, la dangerosité, l'emportait déjà sur la séduction dans nombre d'exemples anciens. (Grandordy 13)

Si elle a l'apparence d'une femme, elle n'en est pas pour autant humaine. Elle est une créature mystérieuse, mélangeant en elle l'animalité et le divin.

Elle est bien sûr, avant toute chose, la femme fatale-à-l'homme, incarnant le destin de l'humanité masculine sacrifiée sur l'autel de l'Espèce. Elle attend sa proie dans l'ombre, avec une tranquillité proprement divine. Elle donne la mort, mais elle est aussi montrée comme un cadavre vivant, charogne repoussante.

(Dottin-Orsini 17-18)

Si son apparence est féminine, elle n'est pas une incarnation de la féminité. Elle est d'ailleurs, la plupart du temps, stérile : "consequently, it is appropriate that the femme fatale is represented as the antithesis of the maternal-sterile or barren, she produces nothing in a society which fetishizes production. » (Doane 2)

La définition la plus concise de la femme fatale est celle de Mary Ann Doane pour qui sa principale caractéristique est de ne pas être ce qu'elle semble être : 
«For the most striking characteristic, perhaps, is the fact that she never really is what she seems to be. »(1).

Milady, dans le roman d'Alexandre Dumas, revêt tout à tour, ces différentes caractéristiques. Sa première apparition a lieu dans le premier chapitre où d'Artagnan la voit discuter avec un homme. C'est tout d'abord la jeunesse et la beauté de Milady qui sont mises en avant :

Son interlocutrice [Milady], dont la tête apparaissait encadrée par la portière, était une femme de vingt à vingt-deux ans. Nous avons déjà dit avec quelle rapidité d'investigation d'Artagnan embrassait toute une physionomie ; il vit donc du premier coup d'œil que la femme était jeune et belle. Or cette beauté le frappa d'autant plus qu'elle était parfaitement étrangère aux pays méridionaux que jusque-là d'Artagnan avait habités. C'était une pâle et blonde personne, aux longs cheveux bouclés tombant sur ses épaules, aux grands yeux bleus languissants, aux lèvres rosées et aux mains d'albâtre. (Dumas 24-25)

La description est celle d'une physionomie possédant tous les atouts de l'innocence. Milady est alors une image unidimensionnelle, sans caractéristique autre que sa beauté. En construisant le personnage de Milady, Alexandre Dumas pervertit progressivement cette première image, en faisant tour à tour de Milady un animal, un être démoniaque, ou un objet:

$[\ldots]$ il recula jusqu'à la ruelle, comme il eût fait à l'approche d'un serpent qui eût rampé vers lui. (Dumas 536)

D'Artagnan s'est fait passer pour de Wardes, pour lequel elle avait une de ces fantaisies de tigresse, indomptable comme en ont les femmes de ce caractère. (Dumas 715)

Il prêtait à cette femme, qui lui apparaissait pareille à un démon, des auxiliaires surnaturels comme elle. (Dumas 675)

Ses lèvres étaient froides : il sembla à d'Artagnan qu'il venait d'embrasser une statue. (Dumas 526)

Ces différentes descriptions déshumanisent petit à petit la figure de Milady, et la rapprochent de la « femme fatale ». Conformément à la définition de Mary Ann Doane, elle sait se faire passer pour ce qu'elle n'est pas, gagnant ainsi la confiance d'autrui :

Alors, comme pour se rendre compte à elle-même des changements qu'elle pouvait imposer à sa physionomie si expressive et si mobile, elle lui fit prendre à la fois toutes les expressions, depuis celle de la colère qui crispait ses traits, jusqu'à celle du plus doux, du plus affectueux et du plus séduisant sourire. (Dumas 717)

Milady représente un danger pour le sexe masculin puisqu'elle outrepasse les barrières de son sexe, en oubliant elle-même sa nature de femme. 
Et cependant, si elle était un homme, elle tenterait tout cela, et peut-être réussiraitelle : pourquoi donc le Ciel s'est-il ainsi trompé, en mettant cette âme virile dans ce corps frêle et délicat! (Dumas 716-717)

Pour la première fois, un mouvement de terreur lui rappela qu'elle était femme. (Dumas 790)

Milady est bien "the antithesis of the maternal », car elle est une mauvaise mère. Le récit d'Alexandre Dumas apprend aux lecteurs, à la moitié de l'ouvrage que Milady est la mère d'un enfant (Dumas 462-472). Jamais celui-ci n'apparaît dans l'histoire, et Milady ne sera jamais présentée dans son rôle de mère. Elle transgresse l'ordre établi en vivant sans homme et en ayant une sexualité libre, qui se satisfait de plusieurs amants. Elle n'a pas de place à l'intérieur d'une structure familiale. Si elle n'est pas «biologiquement» stérile, elle l'est symboliquement puisqu'elle n'élève pas son enfant ${ }^{10}$. Milady parcourt son chemin en solitaire, laissant derrière elle des êtres détruits. Elle est improductive, mais aussi destructrice.

Malgré les attributs négatifs de la «femme fatale », Mary Ann Doane insiste sur la possibilité que cette figure ne se contente pas de remplir la tâche pour laquelle elle a été créée, à savoir mettre en garde les hommes contre le danger des femmes non dominées: "Nevertheless, the representation -like any representation- is not totally under the control of its producers and, once disseminated, comes to take on a life of its own » (3).

Le personnage peut ainsi échapper à son créateur. C'est ce qui arrive à Milady dans Milady de Winter. Émancipée de l'ouvrage d'Alexandre Dumas, elle devient un autre personnage. La déshumanisation progressive de Milady, au fil de l'histoire, n'est plus la révélation de sa « vraie » nature, mais le résultat des actions des hommes sur sa personne. Elle n'est plus une femme inhumaine, on lui prend son humanité. Elle n'est plus un être démoniaque ou un animal, elle est une femme traquée. Dans Milady de Winter, Milady n'est plus une femme fatale aux hommes, mais une femme qui se défend contre eux et qui essaye de se créer une place où vivre, entreprise dans laquelle elle échoue.

Redonner une vie qui lui soit propre à une figure de "femme fatale » est la démarche entreprise par Agnès Maupré dans Milady de Winter. Cette démarche se révèle dès l'abord, dans les couvertures des deux tomes de Milady de Winter

${ }^{10}$ Le fils de Milady apparait dans la suite du roman Les Trois mousquetaires écrite en 1845 et nommée Vingt ans après. Son nom est Mordaunt. Durant l'ouvrage, il essaye de venger sa mère en s'en prenant aux quatre mousquetaires, après avoir tué le Bourreau de Lille. Il meurt en se noyant, refusant de saisir la main que lui tend Athos pour le sauver. 
(Figures 4 \& 5).

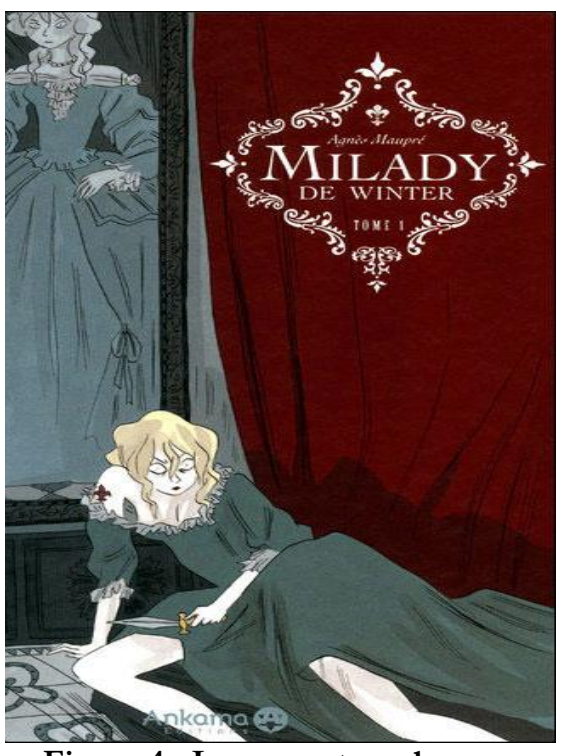

Figure 4 : La couverture du

Tome 1 de Milady de

Winter. CMaupré/Ankama Éditions

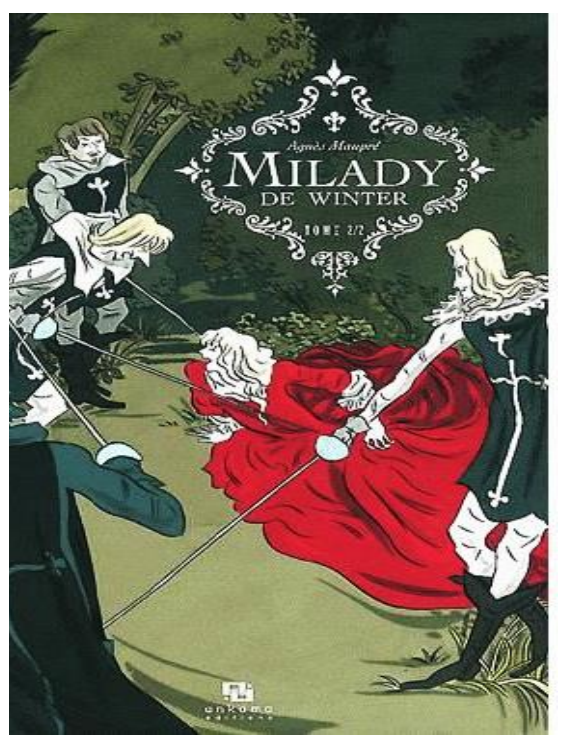

Figure 5 : La couverture du tome 2 de Milady de Winter. CMaupré/ Ankama Éditions

Dans un premier temps (Figure 4) Agnès Maupré transforme l'image archétypale de femme qu'est Milady de Winter en un personnage possédant son propre vécu et ses blessures. La couverture du premier tome présente le personnage de Milady un couteau à la main. L'expression oscille entre l'effarement et l'agressivité. Le couteau lui confère une certaine pugnacité, mais sa pose est celle d'un être acculé. Elle se trouve au pied d'un tableau où elle est représentée avec un air froid et dur. Cette image de Milady, qu'elle possède aujourd'hui dans la culture populaire, tranche avec la femme qui se trouve à terre. Dans un second temps (Figure 5), l'auteure présente Milady encerclée par des hommes. Elle se trouve à terre et désarmée alors qu'ils la menacent avec leur épée. La couleur rouge de sa robe la dissocie du monde qui l'entoure, composé de personnages masculins. Milady n'est pas à sa place dans cette histoire d'hommes. Mais elle est seule et c'est vainement qu'elle crie.

C'est ainsi une réappropriation globale du personnage de Milady de Winter qu'Agnès Maupré effectue dans ses albums :

J'ai rêvassé, fantasmé, avec l'histoire de Dumas comme filet. Il fallait inventer un personnage dont on ne voyait que des petits bouts, donner une cohérence globale. Au bout d'un moment, un personnage, c'est un ami. On sait ce qu'il dirait, ce qu'il ferait dans une situation ou une autre. (Bonadè) 
Agnès Maupré vient défendre Milady face à Alexandre Dumas et aux mousquetaires. Alors que Milady est accusée d'avoir une sexualité trop masculine, Agnès Maupré met en scène une femme avec ses propres désirs. Elle aborde également la maternité de Milady et l'évolution de ses rapports et de son affection pour son fils. Les meurtres ou tentatives de meurtre de Milady ne sont ni occultés ni excusés, mais trouvent une explication, que le lecteur trouvera ou non satisfaisante. Milady les commet chaque fois qu'elle se sent acculée, et que, seule contre tous, elle ne sait comment s'en sortir. Agnès Maupré présente d'ailleurs les remords de Milady, au moment où elle empoisonne Madame Bonacieux. Il ne s'agit pas, à l'inverse du livre, d'une vengeance contre d'Artagnan: «Ah ! ce n'est pas ainsi que je voulais me venger, dit Milady en reposant avec un sourire infernal le verre sur la table, mais, ma foi! on fait ce qu’on peut. » (Dumas 853)

Agnès Maupré lui offre ainsi un passé qui n'existait qu'à l'état de potentialité dans le roman d'Alexandre Dumas. Milady de Winter questionne donc le sens d'une figure dans une époque, mais aussi l'héritage de cette figure pour des lecteurs contemporains. Ce n'est pas uniquement face à Alexandre Dumas, mais aussi face à un imaginaire qui a si facilement transformé Milady en femme froide et dangereuse ${ }^{11}$ qu'Agnès Maupré défend le personnage :

Écrire sur un personnage fictionnel féminin n'était pas le moteur du livre. C'est l'étonnement, en lisant le roman sur le tard, de constater à quel point l'œuvre de Dumas était loin de l'image que j'en avais par les films [...] et particulièrement le personnage de Milady. Après, bien sûr, qu'un personnage aussi complexe et nuancé soit devenu une méchante monolithique dans l'imaginaire collectif, ça interroge un peu mon féminisme. (Bonadè)

\section{Conclusion}

Agnès Maupré, dans Milady de Winter, se réapproprie un personnage féminin historique, et transforme son histoire fictive en une lutte pour le droit à l'indépendance et pour sa propre survie. L'auteure poursuit cette démarche de réappropriation dans un blogue (Figures de proues) où elle écrit des fragments de récits autour de personnages féminins archétypaux, mythiques ou de la culture populaire. Elle leur donne une seconde vie et essaye d'étudier leurs significations à l'époque contemporaine.

${ }^{11}$ Il est d'ailleurs intéressant de voir qu'une critique dithyrambique du premier tome de Milady de Winter commence de la façon suivante :

«Pardonnez-moi cette réjouissante familiarité : Milady de Winter est vraiment la plus grande salope de la littérature, de celles que l'on adore détester, dont l'action néfaste est d'une terrible efficacité, elle parvient à séduire D'Artagnan, à survivre au mousquetaire Athos, alors son mari, à se faire engrosser par le Duc de Buckingham ». 
D'autres nouvelles auteures, arrivées dans l'univers de la bande dessinée ces dernières années, travaillent autour de figures féminines littéraires, historiques ou mythologiques. Anne Simons a publié Persephone aux enfers (Simon), Catherine Meurisse a écrit et dessiné Moderne Olympia (Meurisse). Catel Muller a publié deux bandes destinées historiques centrées sur des femmes, Kiki de Montparnasse et Olympe de Gouges. Contrairement à Milady de Winter dont le personnage central est fictif, les récits de Catel Muller racontent la vie de personnes ayant existé : l'artiste et modèle Kiki de Montparnasse et l'auteure révolutionnaire Olympe de Gouges. Ces bandes dessinées sont inféodées aux sources historiques et proposent, en fin d'album, des biographies, chronologies et bibliographies. Il est cependant possible de dégager un but commun à ces deux démarches différentes : mettre en lumière la place des femmes dans l'histoire et dans l'art. Ces bandes dessinées donnent à lire une histoire des femmes et de leurs représentations, hantée par la domination masculine, mais qui n'est néanmoins pas l'histoire d'une absence. Les femmes ont été présentes et représentées dans l'art, et ces nouvelles auteures de bandes dessinées ${ }^{12}$ ont su en faire, non l'objet de leurs livres, mais leur sujet.

Faut-il voir dans ce mouvement une action politique et féministe, autant qu'esthétique? À cette question, Agnès Maupré répondait de la manière suivante: «Quand je suis rentrée aux Beaux-arts, j'ai eu une prof qui disait que s'inscrire aux Beaux-arts était un acte politique. J'ai trouvé ça idiot. Maintenant moins. Quand on fait des livres, on a la responsabilité de ne pas rendre le monde plus bête qu'il n'est. » (Bonadè)

Il y a ainsi dans Milady de Winter une dimension politique et féministe qui s'inscrit dans la lignée des études de genres. Le travail d'Agnès Maupré, en réécrivant Les Trois mousquetaires, n'est pas tant d'ajouter des nouvelles thématiques à l'ouvrage de Dumas. Elle met plutôt en lumière des éléments déjà présents dans le roman, en leur donnant davantage d'importance.

En se réappropriant une figure culturelle, l'auteure engage les lecteurs à se questionner sur leur interprétation de l'œuvre originale. Les productions culturelles ont davantage été pensées par et pour les hommes - et histoire de l'art a achevé ce travail en occultant les créatrices -, mais la culture appartient aussi à ceux qui la consomment et se l'approprient. S'il existe une grille de lecture dominante des productions culturelles, il reste possible pour les consommateurs de les penser à contre-courant.

${ }^{12}$ Certains hommes se sont aussi joints à ce mouvement, par exemple José-Louis Bocquet qui travaille avec Catel Muller. 


\section{Bibliographie}

Berthelon, Thomas. «Agnès Maupré ("Milady de Winter") : "Après ce qu'elle a vécu, Milady ne peut pas être une méchante absolue.” Actua BD. Web. 30 mai 2015.

Bonadè, Sophie. «Entretien avec Agnès Maupré. ». 9 juin 2015, publié dans cet article.

Boy, Florie, Les femmes dans la bande dessinée d'auteur depuis 1970. Itinéraires croisés: Claire Bretécher, Chantal Montellier et Marjane Satrapi., Mémoire de seconde année, Université de Lyon 2, sous la direction de Sophie Chauveau, juin 2009. Web. 30 mai 2015.

Braun, Heather. The rise and fall of the femme fatale in British literature, 1790-1910. Madison: Fairleigh Dickinson University Press, 2012.

Delphy, Christine. L'ennemi principal 2, Penser le genre. Paris: Éd. Syllepse, 2008.

Doane, Mary Ann. Femmes fatales: Feminism, Film Theory, Psychoanalysis. New York: Routledge, 1991.

Dottin-Orsini, Mireille. Cette femme qu'ils disent fatale: textes et images de la misogynie fin-de-siècle. Paris : Grasset, 1993.

Duby, Georges, et Michelle Perrot. Histoire des femmes en Occident. tome 3 : XVIeXVIIIe siècle. Paris: Académique Perrin Éditions, 2002.

- Histoire des femmes en Occident. tome 4: Le XIXe siècle. Paris: Académique Perrin Éditions, 2002.

Dumas, Alexandre. Les Trois Mousquetaires. Paris: Pocket, 2013.

Dumas, Alexandre et Auguste Maquet. La jeunesse des mousquetaires. Paris : Dufour et Mulat, 1849.

États Généraux de la Bande Dessinée, «Enquête auteurs 2016 : résultats statistiques ». Janvier 2016. Web. 8 février 2016.

Faludi, Susan. Backlash: La guerre froide contre les femmes. Paris: Éditions des Femmes, 1993.

Grandordy, Béatrice, La femme fatale: ses origines et sa parentèle dans la modernité. Paris: L'Harmattan, 2013.

Thierry Groensteen, «Femme (2) : la création au féminin, » Groensteen Thierry (ed.) Dictionnaire esthétique et thématique de la bande dessinée. 2014. Web. 8 février 2016.

Le Roy Ladurie, Irène, « Angoulême: Où sont les femmes? Notes de festival. » Hypotheses.org. Web. 8 février 2016.

Maupré, Agnès. Milady de Winter. Tome 1. Roubaix : Ankama éditions, 2010. . Milady de Winter, Tome 2. Roubaix: Ankama éditions, 2012.

Naeco, Sébastien, «Le tragique printemps de Milady de Winter, » Le comptoir de la BD. 24 novembre 2010. Web. 8 février 2016.

Romito, Patrizia. Un silence de mortes: la violence masculine occultée. Trad. par Jacqueline Julien. 1 vol. Paris: Éd. Syllepse, 2006. 
Stoltenberg, John et al. Refuser d'être un homme: Pour en finir avec la virilité. Paris; Mont-Royal (Québec): Éditions Syllepse, 2013. 
Annexe : Entretien avec Agnès Maupré réalisé le 9 juin 2015 par Sophie Bonadè

Milady de Winter propose de raconter la vie de l'ennemie des mousquetaires dans le roman Les Trois mousquetaires. Pourquoi avoir décidé d'écrire sur un personnage féminin fictionnel?

Écrire sur un personnage fictionnel féminin n'était pas le moteur du livre. Plutôt l'étonnement, en lisant le roman sur le tard, de constater à quel point l'œuvre de Dumas était loin de l'image que j'en avais par les films et autres et particulièrement le personnage de Milady. Après, bien sûr, qu'un personnage aussi complexe et nuancé soit devenu une méchante monolithique dans l'imaginaire collectif, ça interroge un peu mon féminisme.

Avez-vous déjà voulu écrire sur un personnage féminin ayant existé ?

Je fonctionne au coup de cœur. Là j'ai terminé un livre sur le chevalier d'Eon, qui est un personnage qui a existé et qui a été femme un peu. Mais j'aime bien mes personnages d'hommes dans ce livre: Louis XV, Ferrers, du Barry. J'aime autant parler des hommes que des femmes.

La maternité de Milady n'est jamais racontée dans Les Trois mousquetaires. Son fils apparaît surtout dans Vingt ans après. Pourquoi avoir décidé de la mettre en scène?

Je n'avais pas d'enfants quand j'ai écrit Milady et je ne savais pas si j'en voulais. Du coup, ça me paraissait un point intéressant à aborder: qu'est-ce qu'être mère pour quelqu'un qui n'est pas maternel et dont la vie autorise peu de tendresse? La maternité, ça peut être catastrophique, mais difficilement anodin, dans une vie.

Milady, dans le livre et dans votre bande dessinée, est une femme qui a subi de grandes violences physiques et morales (viol, tentative de meurtre). Voulez-vous, dans Milady de Winter, aborder la question des violences faites aux femmes? 
Même quand une femme ne subit aucune violence dans sa vie, il y a des moments où elle ressent son infériorité physique par rapport aux hommes, le soir, dans des rues sombres ou autre. Milady a vécu un copieux échantillonnage de ce qui peut arriver de plus brutal à une femme. Elle rebondit, elle en fait sa force, mais cette force la rend seule de son espèce. Les mousquetaires sont quatre. Elle est seule. Ses actes sont condamnés plus durement que leurs équivalents commis par des hommes. Les mousquetaires sont des soldats, ils sont censés se battre. Chez elle, c'est du vice.

Comment avez-vous réinterprété les événements de la vie de Milady qui ne sont mentionnés que rapidement (et par des hommes) comme l'empoisonnement de Lord de Winter ou la relation de Milady avec le duc de Buckingham ?

J'ai rêvassé, fantasmé, avec l'histoire de Dumas comme filet. Il fallait inventer un personnage dont on ne voyait que des petits bouts, donner une cohérence globale. Au bout d'un moment, un personnage, c'est un ami. On sait ce qu'il dirait, ce qu'il ferait dans une situation ou une autre.

Vous venez de créer un blogue (https://figuresdeproues.wordpress.com/page/2/) où vous travaillez sur de nombreuses figures féminines de la littérature. Quel est votre rapport à ces figures qui ont, pour beaucoup, été l'incarnation des peurs des hommes vis-à-vis de l'indépendance des femmes ? Peuvent-elles être réactualisées ou faut-il en créer de nouvelles?

Les premiers textes de ce blogue ont été écrits pendant un workshop avec Anne Kawala, qui travaille pas mal sur le féminisme. J'aime bien le format, ça permet des mini-portraits. Oui, ça parle d'images de femmes dans l'inconscient collectif, de leur rapport aux hommes. Mais je pense que la version mâle serait aussi intéressante à aborder. J'y songe pour quand $j$ 'aurais un peu de temps. Ce sont des figures qui existeront toujours. C'est le charme des mythes. Ce qui n'empêche pas d'en créer de nouvelles... 


\section{Est-ce que vous inscrivez votre travail dans une démarche politique et/ou féministe?}

Quand je suis rentrée aux beaux-arts, j'ai eu une prof qui disait que s'inscrire aux beaux-arts était un acte politique. J'ai trouvé ça idiot. Maintenant moins. Quand on fait des livres, on a la responsabilité de ne pas rendre le monde plus bête qu'il n'est. Et c'est sûr que le féminisme m'intéresse. J'ai été élevée dans l'idée qu'être une fille ou un garçon ne changeait rien. J'ai eu quelques surprises en avançant en âge.

Pour savoir comment vous nommer dans l'article, préférez-vous le terme d'auteur, d'auteure ou d'autrice?

Auteur, ça me va. Je n'ai pas besoin que tout ce qui me concerne soit féminin. Mais auteure ça marche aussi! 